\title{
PERINATAL MORTALITY RATE IN MULTIPLE PREGNANCIES
}

\author{
S. METLER, W. MIERZEJEWSKI, St. METLER, J. RUDZINSKI \\ Department of Obstetrics and Gynecology, Academy of Medicine, Danzig, Poland
}

The perinatal mortality rate in multiple pregnancies bas been examined both in literature data (where it reaches an average value of $17.5 \%$ ), and on a sample of 619 twin deliveries from the Danzig population.

The perinatal mortality rate in multiple pregnancy may even widely vary, especially according to the period considered, as can be seen from Table 1, collecting the available data from the literature. In the average, over a total of 16,285 deliveries considered for the various periods, it appears to be $17.5 \%$.

Table 2 summarizes our own observations on perinatal mortality in multiple pregnancies, based on a sample of 619 twin deliveries, representing $8.9 \%$ (1:112) over a total of 69,102 general deliveries occurred in our Department of Obstetrics and Gynecology in Danzig in the period 1953-1972.

Our data stress the need for early diagnosis of twin pregnancy, suitable treatment of the patient, and possibly early hospitalization, which will allow for a decrease of premature deliveries, and thus of the perinatal mortality rate, in multiple pregnancies.

Prof. S. Metler, Department of Obstetrics and Gynecology, Academy of Medicine, ul. Kliniczna 1a, 80-402 Gdansk, Poland. 
Table 1. Perinatal Mortality Rate in Multiple Pregnancy (Literature data)

\begin{tabular}{|c|c|c|c|}
\hline Source & Period & $N$ & $\%$ \\
\hline Potter and Grunden 1941 & 1931-1941 & 332 & 13.8 \\
\hline Mummel and Taylor 1946 & $1934-1944$ & 135 & 28.0 \\
\hline Hawker and Allen 1949 & $1937-1947$ & 145 & 22.0 \\
\hline Potter and Fuller 1949 & $1941-1947$ & 252 & 14.0 \\
\hline Bender 1952 & $1946-1951$ & 472 & 11.0 \\
\hline Fink 1955 & 1955 & 669 & 19.3 \\
\hline Anderson 1956 & $1938-1942$ & 172 & 23.2 \\
\hline Anderson 1956 & $1934-1947$ & 262 & 20.2 \\
\hline Anderson 1956 & $1948-1952$ & 360 & 15.5 \\
\hline Guttmacher and Kohl 1958, 1962 & $1950-1956$ & 1327 & 13.3 \\
\hline Little and Friedman 1958 & $1947-1957$ & 417 & 9.2 \\
\hline Danielson 1960 & $1940-1957$ & 1006 & 12.0 \\
\hline Weddel et al. 1960 & & 203 & 10.0 \\
\hline Aaron et al. 1961 & & 204 & 10.3 \\
\hline Bach and Kiffe 1962 & & 255 & 18.8 \\
\hline Graves et al. 1962 & & 872 & 14.4 \\
\hline Mac Donald 1962 & & 500 & 12.5 \\
\hline Spurway 1962 & & 353 & 12.2 \\
\hline Camilleri 1963 & & 4300 & 11.6 \\
\hline Heery 1963 & $1946-1951$ & 226 & 13.3 \\
\hline Robertson 1964 & $1956-1962$ & 496 & 14.0 \\
\hline Sparling 1967 & $1944-1962$ & 192 & 14.0 \\
\hline Holtorff 1964 & $1951-1962$ & 254 & 18.9 \\
\hline Eckart and Spenke 1970 & & 268 & 12.1 \\
\hline Gizowski 1935 & $1915-1925$ & 174 & 23.6 \\
\hline Krasucka and Zalewska 1957 & $1951-1956$ & 234 & 23.0 \\
\hline Sternadel & $1923-1962$ & 740 & 28.0 \\
\hline Slomko and Kuczynski $1965 a$ & $1958-1964$ & 467 & 13.4 \\
\hline Sternadel & $1963-1965$ & 42 & 11.8 \\
\hline Metler et al. (present research) & 1953-1972 & 619 & 21.8 \\
\hline Total & & 16285 & 17.5 \\
\hline
\end{tabular}

Table 2. Perinatal Mortality Rate and Birth Weight in Multiple Pregnancy (Personal observation)

\begin{tabular}{|c|c|c|c|c|c|c|c|c|c|c|c|}
\hline \multirow[b]{2}{*}{$\begin{array}{l}\text { Total no. } \\
\text { of deliveries }\end{array}$} & \multicolumn{2}{|c|}{ 1953-1957 } & \multicolumn{2}{|c|}{$1958-1962$} & \multicolumn{2}{|c|}{$1963-1967$} & \multicolumn{2}{|c|}{$1968-1972$} & \multicolumn{3}{|l|}{ 'Total } \\
\hline & 184 & & 122 & & 127 & & 186 & & 619 & & \\
\hline Birth weight & $\begin{array}{l}\text { Live- } \\
\text { born }\end{array}$ & $\begin{array}{l}\text { Dead } \\
\text { after } \\
\text { delivery }\end{array}$ & $\begin{array}{l}\text { Live- } \\
\text { born }\end{array}$ & $\begin{array}{l}\text { Dead } \\
\text { after } \\
\text { delivery }\end{array}$ & $\begin{array}{l}\text { Live- } \\
\text { born }\end{array}$ & $\begin{array}{l}\text { Dead } \\
\text { after } \\
\text { delivery }\end{array}$ & $\begin{array}{l}\text { Live- } \\
\text { born }\end{array}$ & $\begin{array}{l}\text { Dead } \\
\text { after } \\
\text { delivery }\end{array}$ & $\begin{array}{l}\text { Live- } \\
\text { born }\end{array}$ & $\begin{array}{l}\text { Dead } \\
\text { after } \\
\text { delivery }\end{array}$ & $\%$ \\
\hline $\begin{array}{l}<1000 \\
1001-1500 \\
1501-2000 \\
2001-2500 \\
2501-3000 \\
>3000\end{array}$ & $\begin{array}{r}42 \\
20 \\
52 \\
106 \\
96 \\
52\end{array}$ & $\begin{array}{r}39 \\
13 \\
12 \\
13 \\
3 \\
0\end{array}$ & $\begin{array}{l}26 \\
20 \\
34 \\
62 \\
56 \\
46\end{array}$ & $\begin{array}{r}24 \\
14 \\
7 \\
4 \\
1 \\
0\end{array}$ & $\begin{array}{l}26 \\
20 \\
36 \\
70 \\
66 \\
36\end{array}$ & $\begin{array}{r}25 \\
13 \\
5 \\
5 \\
2 \\
0\end{array}$ & $\begin{array}{l}54 \\
24 \\
64 \\
98 \\
76 \\
56\end{array}$ & $\begin{array}{r}54 \\
21 \\
9 \\
4 \\
2 \\
1\end{array}$ & $\begin{array}{r}148 \\
84 \\
186 \\
336 \\
294 \\
190\end{array}$ & $\begin{array}{r}142 \\
61 \\
33 \\
26 \\
8 \\
1\end{array}$ & $\left.\begin{array}{r}95.9 \\
72.6 \\
17.7 \\
7.7 \\
2.7 \\
0.5\end{array}\right\} 6.7$ \\
\hline Total & 368 & 80 & 214 & 50 & 254 & 50 & 372 & 95 & 1238 & 271 & 21.9 \\
\hline $\begin{array}{l}\text { Prenatal deaths } \\
\text { Intranatal deaths } \\
\text { Postnatal deaths } \\
\text { All causes }\end{array}$ & $\begin{array}{l}12 \\
17 \\
47 \\
76\end{array}$ & & $\begin{array}{r}3 \\
4 \\
42 \\
49\end{array}$ & & $\begin{array}{r}7 \\
5 \\
37 \\
49\end{array}$ & & $\begin{array}{r}17 \\
7 \\
72 \\
96\end{array}$ & & $\begin{array}{r}39 \\
33 \\
198 \\
270\end{array}$ & $\begin{array}{r}3.1 \\
2.7 \\
16.0 \\
21.8\end{array}$ & \\
\hline
\end{tabular}

